Gerión. Revista de Historia Antigua

ISSN: 0213-0181

\title{
Considerazioni sulla paternità della raccolta priscillianista di Würzburg
}

\author{
Maria Veronese ${ }^{1}$
}

Recibido: 15 de febrero de 2021 / Aceptado: 8 de marzo de 2021

Riassunto. Il saggio analizza le citazioni bibliche e le formule di introduzione delle citazioni negli scritti della raccolta priscillianista di Würzburg per evidenziare analogie e differenze che possano fornire qualche indizio utile alla definizione della paternità della raccolta. Contro le conclusioni dell'editore Georg Schepss, questa analisi dimostra che i Tract. V e VII si differenziano dai rimanenti Tract. IV, VI, IX e X, mentre il Tract. VIII fa causa a sé; più complicati risultano invece i rapporti tra i Tractatus e le tre apologie iniziali.

Parole chiave: Priscilliano; citazioni bibliche; formule introduttive; Vetus Latina.

\section{[en] Some Considerations on the Authorship of the Priscillianist Collection from Würzburg}

\begin{abstract}
The paper analyses the biblical quotations and the formulas for introducing quotations in the writings of the Würzburg Priscillianist corpus in order to highlight similarities and differences; the results may provide us with some useful clues in defining the authorship of the collection. Against the conclusions of the first editor Georg Schepss, this analysis shows that the Tract. V and VII differ from the Tract. IV, VI, IX and X, while the Tract. VIII is isolated; more intricate is the relationship between the Tractatus and the three apologetic writings.
\end{abstract}

Keywords: Priscillian; Biblical Quotations; Introductory Formulas; Vetus Latina.

Sommario. 1. La raccolta priscillianista di Würzburg. 2. Status quaestionis. 3. Formule di introduzione delle citazioni bibliche. 4. Citazioni bibliche 5. Conclusioni. 6. Bibliografia.

Cómo citar: Veronese, M. (2021): Considerazioni sulla paternità della raccolta priscillianista di Würzburg, en Gerión 39(2), 485-501.

1 Università di Padova.

E-mail: maria.veronese.1@unipd.it

ORCID: 0000-0002-4259-6653 
Le fonti più antiche tramandano un ritratto di Priscilliano come discendente di una nobile famiglia, persona molto colta e perfettamente istruita, autore di molti scritti, ${ }^{2}$ un ritratto che coincide con quanto si afferma nel Liber apologeticus, il primo testo della raccolta di Würzburg, il cui autore si dichiara discendente di una nobile famiglia e dotato di una certa cultura (non ita obscuro editi ad saeculum loco aut insipientes vocati sumus $u t \ldots) .^{3}$

Questo ritratto e la notizia geronimiana sui multa opuscula da lui composti, combinati all'ampia diffusione del movimento priscillianista, hanno fatto sì che un gran numero di scritti venisse attribuito direttamente a Priscilliano o a qualche suo seguace; al suo nome sono state infatti indiscriminatamente ricondotte numerose opere sulla base della reale o presunta origine ispanica, della presenza di sviluppi teologici eterodossi, dell'utilizzo di apocrifi o dell'invito a un ascetismo rigoroso. ${ }^{4}$ Ma l'unica opera la cui attribuzione a Priscilliano è sicura e confermata dal prologo sono i Canones in Pauli apostoli epistulas, che subirono una revisione in senso ortodosso, la cui portata non è facilmente individuabile, da parte di un non meglio identificato Peregrinus episcopus. ${ }^{5}$

In questa sede intendo affrontare la questione relativa alla paternità degli undici Tractatus anonimi (CPL 785), scoperti alla fine del XIX secolo e pubblicati nel 1889 sotto il nome di Priscilliano nel diciottesimo volume del Corpus Vindobonense da Georg Schepss, prestando particolare attenzione alle citazioni bibliche.

\section{La raccolta priscillianista di Würzburg}

Il manoscritto della Universitätsbibliothek di Würzburg (M.p. th. q. 3), ${ }^{6}$ datato alla fine del secolo $\mathrm{V}$, vergato in scrittura onciale, presumibilmente di origine italiana, è l'unico testimone a tramandare questi undici componimenti senza fornire alcuna indicazione circa l'autore. Si tratta nell'ordine di tre apologie, sette sermoni ${ }^{7}$ e una preghiera finale.

La prima delle tre apologie, generalmente indicata come Liber apologeticus (= Tract. I) ${ }^{8}$ è indirizzata a un gruppo di sacerdoti da una persona che, a nome di una comunità di confratelli, avverte la necessità di presentare ai beatissimi sacerdotes un documento

2 Hier. Vir.Ill. 121: edidit multa opuscula; Sulp. Sev. Chron. 2.46.3: familia nobilis, predives opibus... multa lectione eruditus. Tutte le date in questo articolo sono A.D. salvo diversa indicazione.

Lib. apol. I (CSEL 18.4.9-10).

Cf. CPL 788-796c; 1872; 2302; CPPM IIA, 1510-1513; 2902.

Nel proemio dell'opera un certo Peregrinus episcopus conferma che questo scritto va attribuito a Priscilliano, anche se molti lo avevano posto sotto il nome di Gerolamo, la cui autorità garantì all'opera vasta diffusione. Nell'attribuirlo a Priscilliano Peregrinus aggiunge anche di avere corretto in senso ortodosso alcune espressioni scritte pravo sensu (Proemium Peregrini, CSEL 18.109.6). Quanto peso abbia avuto questo intervento, è difficile dire, ma gli studiosi sono concordi nel ritenere che Peregrinus non abbia modificato sostanzialmente il contenuto dell'opera, ma sia piuttosto intervenuto sul testo biblico emendandolo sulla base della Vulgata geronimiana; cf. De Bruyne 1914-1919, 384-385; Fischer 1963, 532-540; Vollmann 1974, 552; Chadwick 1976, 60; è stata unanimemente rigettata dalla critica l'ipotesi di D’Alès (1936, 152-153, n. 1) di identificare Peregrinus con Bachiario.

6 Thurn 1984, 87-88; CLA IX 1431; Schepss 1889, IX-XXVIII. La digitalizzazione del manoscritto è consultabile al seguente link: http://vb.uni-wuerzburg.de/ub/mpthq3/pages/mpthq3/3.html.

7 Questi sette testi sono gli unici che a buon diritto si possono definire tractatus, nel senso di sermone a commento di un testo biblico (cf. Bardy 1946; Mohrmann 1961, 70-71); si vedano le frequenti invocazioni a dilectissimi fratres o dilectissimi mihi o dilectissimi in deo; cf. ad esempio Tract. IV (CSEL 18.58.6 e 24); Tract. V (CSEL 18.64.4; 67.7); Tract. VII (CSEL 18.85.3). Sia nell'edizione di Schepss (CSEL 18 [1889]) sia in quella di Marco Conti (2010) tutti i testi sono intitolati Tractatus e numerati da I a XI.

8 In realtà il manoscritto non riporta alcun titolo. 
che condanni le dottrine eretiche contrarie a Cristo e professi, invece, la fede in Cristo. Nonostante sia già nota a tutti la nostra fides, grazie al libello dei confratelli Tiberiano, Asarbio ${ }^{9}$ e altri, e grazie alla conoscenza personale e diretta, tuttavia l'autore ripeterà la sua professione di fede; egli non è di umili origini né un illetterato e, dopo essere passato attraverso le vicissitudini della vita, ha ripudiato il mondo per entrare nel porto sicuro della fede: con il battesimo egli ha respinto definitivamente le lusinghe del secolo per dedicarsi completamente a Cristo. Tale descrizione sembra adattarsi perfettamente a Priscilliano. Più difficile è tentare di stabilire la datazione del testo: poiché l'autore si rivolge a beatissimi sacerdotes, si pensa che l'occasione sia stata fornita da un concilio di vescovi, generalmente identificato dagli studiosi con quello di Cesaraugusta del $380,{ }^{10}$ mentre in seguito ha preso piede l'ipotesi che si trattasse del successivo concilio di Bordeaux del $384 .{ }^{11}$

Il Liber ad Damasum episcopum, come il manoscritto intitola quello che nell'edizione di Schepss è il Tractatus II ${ }^{12}$ è anch'esso un'apologia composta a nome di un gruppo di persone e indirizzata a Damaso, vescovo di Roma, la cui compilazione va datata tra la fine del 381 e la primavera del 382, quando i tre vescovi Instanzio, Salviano e Priscilliano furono a Roma per chiarire i fatti seguiti al concilio di Cesaraugusta e per difendersi dalle accuse.

La terza apologia della raccolta, mutila dell'inizio, è introdotta da un incipit che reca: incipit de fide de apocryfis (= Tract. III) ${ }_{13}^{13}$ titolo che fu integrato da Schepss, seguito da Conti, in Liber de fide $<e t>$ de apocryfis; ${ }^{14}{ }^{1}$ 'autore difende e legittima la lettura dei libri apocrifi con solide argomentazioni che sfruttano le testimonianze delle Scritture canoniche.

Il gruppo dei sette sermoni propone l'esegesi di passi veterotestamentari, dove predomina l'esegesi allegorica con una forte tensione parenetica; la disposizione e la scelta dei testi (introduzione sulla Pasqua, Gn 1, Ex 12, Pss 1, 3, 14 (?), ${ }^{15} 59$ ), introdotti da una dedica che invita una felix Amantia ${ }^{16}$ alla lettura comunitaria e concluso da

9 Gerolamo in Vir.Ill. 123 ricorda un Apologeticum, oggi perduto, scritto da Tiberiano in stile ampolloso e ricercato. Sulpicio Severo (Chron. 2.51.4) cita un diacono Asarivus, che fu condannato con Priscilliano e altri alla pena capitale.

10 Sittl 1889, 44; Hilgenfeld 1892, 76-77; Chadwick 1976, 51.

11 Morin 1913, 157-162, che riprende la tesi di Dierich 1897; per Vollmann (1974, 557-558) può 'forse' trattarsi di questo concilio. Babut $(1909,204-208)$ avanzò l'ipotesi che l'apologia fosse stata composta dopo il concilio di Cesaraugusta, quando, in seguito allo scisma prodottosi nella chiesa di Mérida, di cui parla il Liber ad Damasum, i laici secessionisti dovettero presentare la loro professione di fede per essere riammessi alla comunione della chiesa. Questa tesi è stata più recentemente presa in considerazione da Virginia Burrus (1995, 55), la quale considera il contesto dello scisma di Mérida come il più probabile. Secondo Sanchez (2013) l'opera, da ascrivere a Priscilliano, fu composta in diversi momenti a partire da una raccolta di anatematismi e da una professione di fede, poi riuniti e amalgamati insieme.

12 Si vedano incipit in f. 40v e explicit in f. $55 \mathrm{r}$.

13 Il testo comincia al f. 56r, l'incipit si trova al f. 55r, mentre il verso dello stesso folio è stato lasciato in bianco.

14 Secondo Paret (1891, 182-183 in nota), il fatto che lo scritto, così come ci è pervenuto, entri subito in argomento, dimostra che non una lunga parte del testo è andata perduta; ma egli avanzò anche l'ipotesi che, se la lacuna fosse più ampia, potrebbe essere andato perduto un intero trattato intitolato De fide; così anche Sittl $(1891,268)$. A parere di Hilgenfeld (1892, 71 e 73) e di Vollmann (1974, 558), invece, il titolo indica un unico scritto e va inteso come 'a cosa bisogna prestare fede negli apocrifi' o 'sulla credibilità degli apocrifi'.

15 Dopo il f. 121 è caduto un intero quaternione; tale caduta risale a una data posteriore ai secc. VIII/IX quando fu apposta sul codice una seconda numerazione, cf. Thurn 1984, 87. Considerando che nell'assemblare la raccolta dei trattati si è mantenuto l'ordine dei libri biblici, il Tract. IX deve essere un commento ad un salmo compreso tra il Ps 3 (Tract. VIII) e il Ps 59 (Tract. X). Giovanni Mercati (1901, 129-131) propose due soluzioni: o si tratta della parte finale del Tract. VIII sul Salmo 3, del quale si sarebbe perduta la parte centrale, oppure-ipotesi privilegiata-di un trattato su un salmo a sé stante che va individuato nel Ps 14, come suggerisce il tema centrale del trattato. Tale opinione, sostenuta dalla grande autorità di uno specialista dei salmi quale fu Mercati, è accettata e condivisa da quasi tutti gli studiosi a partire da Bardy (1936, 394), fino a Vollmann (1974, 553), Chadwick $(1976,65)$ e Sanchez $(2009,80)$.

16 Sul f. 74v si legge: lege felix Amantia cum tuis in Christo Iesu Domino nostro; il nome proprio si legge sotto la rasura, mentre in margine è stata apposta una nota tironiana interpretata come Amantius o Amius, cf. Thurn 1984, 87. 
una preghiera di benedizione rivolta ai fedeli (Benedictio super fideles $=$ Tract. XI), fanno pensare che la raccolta di questi sermoni sia stata allestita appositamente come una serie di letture adatte per il periodo della Quaresima. ${ }^{17}$

\section{Status quaestionis}

Nel 1889 l'editio princeps dei trattati di Würzburg suscitò un vivace dibattito tra gli studiosi, che potevano finalmente leggere alcuni scritti che venivano attribuiti all'eresiarca Priscilliano, di cui fino ad allora non si conosceva altro se non ciò che le fonti ortodosse avevano riportato. Le questioni su cui si concentrò la discussione furono principalmente due: la reale o presunta eterodossia di Priscilliano e la paternità della raccolta.

Per diversi decenni la critica continuò a muoversi su due binari contrapposti, vedendo da un lato schierati coloro che, facendo leva sul contenuto sostanzialmente ortodosso degli scritti di Würzburg, rinnovarono l'immagine di Priscilliano considerato come un cristiano fedele ingiustamente condannato dalle autorità ecclesiastiche e civili, e dall'altro coloro che, ritenendoli una raccolta emendata a fini apologetici, mantenevano e confermavano l'immagine tradizionale dell'eretico. Solamente dopo la metà del secolo scorso, con lo studio di Willy Schatz e poi, alla metà degli anni Settanta, con gli studi di Jacques Fontaine e Henry Chadwick, il movimento priscillianista cominciò a essere analizzato alla luce della spiritualità ascetica del IV secolo e della concorrenza tra due concezioni antagoniste della Chiesa: quella ufficiale che difendeva il modello della Chiesa gerarchica e che, intrecciando più stretti rapporti con l'Impero, si presentava sempre più mondanizzata, e quella che propugnava l'ideale di una Chiesa carismatica, pura e scismatica. ${ }^{18}$

La questione sulla paternità ebbe invece minor risonanza rispetto alla precedente, in quanto pressoché tutte le recensioni all'edizione di Schepss lodarono il suo importante e attento lavoro filologico e storico e gli studiosi furono concordi con la sua attribuzione degli undici trattati a Priscilliano, evidenziando che essi rivelano chiaramente un'impronta unitaria. Già tre anni prima della pubblicazione del testo critico, nel 1896 durante una conferenza tenuta all'Università di Würzburg dal titolo "Priscillian, ein neuaufgefundener lateinischer Schriftsteller des vierten Jahrhunderts", Schepss aveva posto all'attenzione del mondo scientifico l'importanza del manoscritto ivi custodito e aveva proposto, sulla base di indizi esterni (notizie storiche coniugate con le notizie del Liber ad Damasum) e interni (in primis lingua e metodo di citazione, ma anche temi e passi paralleli), l'attribuzione degli scritti a un unico autore che non poteva che essere identificato con Priscilliano, ${ }^{19}$ corroborando la sua tesi con un articolo, pubblicato nello stesso anno, sull'analisi linguistica dei trattati. ${ }^{20}$

\footnotetext{
17 Vollmann 1974, 553-554; Veronese 2004, 202.

18 È la tesi principale di Schatz 1957; cf. in particolare cap. 1.4: "Die Gründe des Konflikts: die Polarität von Amt und Charisma, die Spannung zwischen asketischer Sonderorganisation und kirchlicher Gemeindeordnung", 197-259. Cf. anche Fontaine 1981, 196-198.

19 Schepss $(1886 \mathrm{a}, 12)$ dichiara di avere appreso da una lettera del professore norvegese Carl Paul Caspari che circa cinque anni prima un amico di Würzburg (i.e. il bibliotecario Ruland) aveva inviato una copia del manoscritto di Würzburg a Ignaz von Döllinger, il quale avanzò il nome di Priscilliano quale autore.

20 Schepss 1886b, 309-310.
} 
Solo alcuni studiosi avanzarono seri dubbi sulle conclusioni di Schepss: Karl Sittl, Emil Michael e in parte anche Franz Xaver Kraus; ${ }^{21}$ le critiche riguardavano principalmente le pretese analogie di stile e lingua all'interno del corpus di Würzburg, evidenziate dall'editore a discapito delle differenze e trascurando gli elementi caratteristici e singolari che contraddistinguevano alcuni trattati, nonché le presunte somiglianze di lingua e contenuto dei trattati con i Canones in Pauli apostoli epistulas.

Contro le obiezioni di Sittl e Michael, nel 1893 Schepss avvertì la necessità di ritornare sull'argomento per replicare alle loro accuse e riconfermare la propria ipotesi unitaria, presentando nuove analogie linguistiche e stilistiche, comuni riferimenti biblici e modelli patristici. ${ }^{22} \mathrm{Ma}$ in sostanza la sua tesi non risultò pregiudicata dalle accuse e dai dubbi di Sittl e Michael, ${ }^{23}$ mentre cominciò a vacillare quando nel 1913 dom Germain Morin pubblicò un articolo contro l'attribuzione a Priscilliano e a favore di Instanzio, non contestando l'unitarietà d'autore dei trattati, bensì scardinando la certezza di Schepss circa la perfetta analogia di stile e pensiero tra i trattati e i Canones. La tesi di Morin suscitò subito un'ampia eco tra gli studiosi, aprendo un nuovo e vasto dibattito e, considerata l'autorevolezza dello studioso benedettino, essa trovò anche diversi sostenitori, tra cui Adhémar d'Alès, ${ }^{24}$ il quale sottolineò che dai trattati emerge in effetti un priscillianismo sconosciuto, testimone di una tendenza meno nociva interna alla setta stessa.

Nei decenni che seguirono, la critica non si curò più molto della questione della paternità dei trattati editi da Schepss, ma preferì privilegiare altri aspetti del priscillianismo, legati all'origine del monachesimo occidentale e, intorno agli anni Sessanta e Settanta, al ruolo politico e sociale del movimento. Nel 1974 la questione fu riaperta da Benedikt Vollmann, ${ }^{25}$ il quale si schierò decisamente contro l'unitarietà d'autore degli undici trattati di Würzburg, ritenendo che tutti gli studiosi a partire da Schepss avessero commesso due errori di fondo: in primo luogo, che dalla presenza di formulazioni analoghe fossero giunti alla conclusione dell'unitarietà d'autore, escludendo l'ipotesi che potesse trattarsi anche di opere di una scuola; in secondo luogo, che non avessero concentrato l'attenzione sullo stile dei trattati, ma solamente sul vocabolario. Contro l'unitarietà d'autore egli propose di riconoscere diverse mani nei trattati: il Liber apologeticus con molta probabilità non è di Priscilliano, né l'autore è da identificare con lo stesso del Liber ad Damasum, apologia che è stata sicuramente scritta da uno dei tre vescovi che si recarono a Roma o da uno dei loro segretari, e che potrebbe essere stata scritta, dunque, da Priscilliano in persona. Il Liber de fide de apocryfis, giudicato uno scritto assai pregevole sia dal punto di vista formale sia dal punto di vista del contenuto, è quello che meglio si addice alla descrizione di un Priscilliano erudito ed eloquente proposta da Sulpicio Severo. I Tractatus IV-XI fanno parte della vasta letteratura priscillianista prodotta

$21 \quad$ Sittl 1889; 1891; Michael 1892; 1894; Kraus 1891.

22 Schepss 1893, 134-143.

23 Nel 1896 Merkle, presentando una relazione sullo stato della disputa intorno all'autore dei trattati di Würzburg, concluse appoggiando la tesi di Schepss e non esprimendo alcun dubbio sulla paternità di Priscilliano (Merkle 1896, 639). Babut nella sua monografia su Priscilliano, pubblicata nel 1909, attribuì gli scritti a un solo e allo stesso autore: Priscilliano (Babut 1909, 199). Anche Puech nel 1912 non sembrò avere alcun dubbio sulla questione che riguarda l'attendibilità di questa attribuzione.

24 D'Alés 1936, 78. Secondo D'Alés nei trattati è riconoscibile l'impronta di uno stesso autore, ovvero di Instanzio, ma in essi vi è sicuramente anche qualche traccia di Priscilliano (cf. anche D’Alés 1936, 56).

25 Vollmann 1974, 554-559. 
dopo la morte di Priscilliano (385) e vanno anch'essi attribuiti a diverse mani: i Tract. IV, VI, X e forse IX sono scritti in quello che Vollmann definisce il peggiore latino nel quale si sia imbattuto, con uno stile sommamente oscuro, altisonante e pretenzioso; essi appartengono a un unico autore, che non è possibile identificare con Priscilliano, ma che va forse ricercato nell'ambito dei vescovi galiziani; i Tract. V e VII sono probabilmente del medesimo autore, differenziandosi dagli altri per l'assenza di citazioni da Ilario, per lo stile (cola paralleli o doppie antitesi) e per la variata introduzione di citazioni bibliche; il loro contenuto è giudicato assolutamente priscillianista; la Benedictio super fideles, stimata sia stilisticamente sia teologicamente lo scritto di gran lunga migliore della raccolta, costituisce uno scritto a sé stante. Infine il Tract. VIII fa causa a sé, dal momento che non vi si riscontrano alcun concetto né alcuna espressione tipicamente priscillianista, ma solo una lunga serie di peculiarità.

Il contributo di Vollmann non riscosse alcun favore da parte della critica, essendosi imposta nel frattempo l'autorevole opinione di Henry Chadwick, il quale riteneva indubitabile il fatto che il complesso dei trattati emerge dal medesimo ambiente priscillianista; le numerose ripetizioni attestano che unica è la mente che si è messa all'opera; e concludeva: "The main body of the tractates comes from Priscillian himself". ${ }^{26}$ Tutti i trattati sono dunque espressione di un medesimo ambiente culturale e come tali vanno indagati nel loro insieme senza che sia possibile fare distinzioni tra gli scritti di Priscilliano o di qualche altro priscillianista; ${ }^{27}$ una sola eccezione potrebbe essere costituita, secondo Chadwick, dal Tract. V, che contiene scarsi riferimenti agli altri trattati, nonostante i temi che vi sono espressi non siano in contrasto con essi, concludendo che forse è opera di un altro membro della confraternita priscillianista. ${ }^{28}$

Negli ultimi anni sembra invece avere ripreso quota l'ipotesi antiunitaria favorevole ad attribuire gli scritti della raccolta a più autori o perlomeno a individuare all'interno della raccolta dei sottogruppi omogenei. Marco Conti ritiene che le tre apologie che aprono il corpus di Würzburg siano opera di un unico autore da identificare con il leader della setta; per quanto riguarda i Tractatus IV-X propende per l'ipotesi di ascriverli a diversi autori (IV, VI e X a un autore; V e VII ad un altro autore; VIII e IX sono troppo mutili per formulare ipotesi), mentre la Benedictio super fideles è ritenuta opera di Priscilliano. ${ }^{29}$ Anche secondo Sylvain Jean Gabriel Sanchez le analogie tra i primi tre scritti apologetici confermano l'attribuzione a un unico e medesimo autore, ovvero Priscilliano, mentre i rimanenti Tractatus sono suddivisi in due gruppi: i Tract. IV, VI, IX, X e XI sono da datare successivamente al periodo del soggiorno bordolese quando Priscilliano ebbe l'opportunità di conoscere 1'opera di Ilario di Poitiers, mentre i Tract. V, VII e VIII restano di datazione incerta. ${ }^{30}$

A mio avviso vi sono diversi elementi che inducono ad attribuire gli scritti della raccolta di Würzburg a diversi autori. In uno mio studio di qualche anno fa

\footnotetext{
26 Chadwick 1976, 66-69.

27 Su questa linea si collocano anche Orbe (1981, 311) e Burrus (1995, 3 n. 8; 167 n. 8; Burrus 1997, 401 n. 3) la quale si riferisce semplicemente a Priscilliano considerandolo come il più probabile autore dei trattati.

28 Chadwick $(1976,66)$ nota una certa familiarità con il Tract. VIII per quanto riguarda l'insistenza sul tema della responsabilità dell'uomo.

29 Conti 2010, 15-16.

30 Sanchez 2009, 68-77.
} 
sull'utilizzo del De trinitate di Ilario ${ }^{31}$ concludevo, contro Schepss, che le citazioni dell'opera teologica del vescovo gallico non sono presenti in tutti gli undici componimenti, come invece lascia intendere l'indice di CSEL, ma limitati a cinque trattati (Tract. IV, VI, IX e X) e alla Benedictio super fideles; inoltre, analizzando il modo in cui l'autore priscillianista ha ripreso i passi ilariani, dimostravo che talora egli citava Ilario condividendone il pensiero, altre volte si serviva di formulazioni e espressioni ilariane per trasmettere idee e concetti completamente nuovi e del tutto estranei al contesto originale. Questa constatazione mi ha condotto a riconsiderare la questione dell'unitarietà d'autore. Sulla scorta delle ragionevoli osservazioni di Vollmann, anch'io ritengo che nel manoscritto di Würzburg siano confluiti testi di autori diversi, che potrebbero manifestare il pensiero priscillianista in diverse fasi del suo sviluppo, con la probabile eccezione del Tract. VIII che sotto vari aspetti rivela caratteristiche estranee al resto della raccolta. È evidente che per fondare questa ipotesi su prove certe e argomentazioni solide è necessario approfondire il testo degli scritti di Würzburg sotto molteplici prospettive: lingua, stile, contenuto, fonti e modelli. Propongo qui alcune considerazioni limitatamente all'impiego della sacra Scrittura negli scritti del corpus priscillianista, nella convinzione che qualche ulteriore contributo alla questione della paternità d'autore possa essere offerto anche dall'esame delle citazioni bibliche, considerato che nell'ambito del movimento priscillianista la lettura e lo studio della sacra Scrittura costituivano l'attività principale del fedele. ${ }^{32}$

Interessanti elementi di indagine si rivelano sia le formule di introduzione delle citazioni bibliche, sia il testo di alcuni passi biblici per i quali si individuano citazioni ripetute nei trattati di Würzburg.

\section{Formule di introduzione delle citazioni bibliche}

L'esame delle formule utilizzate per introdurre le citazioni bibliche consente di individuare alcune interessanti analogie e alcune significative differenze negli scritti della raccolta di Würzburg. ${ }^{33}$ Dalla presente analisi resta esclusa la Benedictio super fideles che, pur presentando una fitta trama scritturistica, non riporta citazioni bibliche dirette.

Vollmann si era servito dell'esame delle formule introduttive come una delle prove contro l'unitarietà d'autore, rilevando che in alcuni scritti le formule di introduzione sono fisse e non presentano significative varianti, mentre in altri sono più composite e elaborate; egli individuava, dunque, un primo gruppo costituito dai Tract. IV, VI, IX e X, che si differenzia da un secondo costituito dai Tract. V e VII; la medesima discrepanza, secondo la sua analisi, è evidente anche tra il Liber apologeticus e il Liber ad Damasum. ${ }^{34}$

La formula che ritorna con maggiore frequenza è sicut scribtum est (68 occorrenze totali), che si riscontra con particolare insistenza nelle due apologie iniziali (rispettivamente 33 e 12 occorrenze) e nel Tract. V (9 occorrenze); con quest'ultimo

\footnotetext{
Veronese 2003; cf. anche Veronese 2013.

Veronese 2018.

Per una analisi dettagliata, Veronese 2018, 127-138.

Vollmann 1974, 556-557.
} 
testo, inoltre, i primi tre scritti apologetici condividono anche la formula cum scribtum sit (4, 2, 3 occorrenze rispettivamente nelle tre apologie, e 3 nel Tract. V), mentre la relativa (secundum) quod scribtum est si trova 4 volte nel Tract. VI, 3 volte nel Liber apologeticus e 1 nel Tract. IV. La formula sicut 'profeta/apostolus/...' ait, che nel Liber apologeticus ricorre ben 28 volte (contro le 33 di sicut scribtum est), è utilizzata 6 volte nel Liber de fide de apocryfis e nei Tract. VI e X, e ben 3 volte nel breve frammento del Tract. IX. Il Tract. VI rivela anche una predilezione per le espressioni participiali (dicente 'propheta/apostolo/...', 10 ricorrenze), secondo formulazioni ricorrenti anche nelle tre apologie (rispettivamente 8, 4 e 4 ricorrenze) e nei Tract. IV e X (rispettivamente 4 e 6 occorrenze). Le formule particolari sono poche e prevalentemente attestate nel Liber apologeticus e nel Liber de fide de apocryfis, circostanza che è motivata sia dalla numerosità delle citazioni bibliche in questi scritti particolarmente lunghi, sia dal genere letterario che richiedeva per gli scritti apologetici una maggiore ricercatezza e varietà di stile. Per quanto riguarda le formule di passaggio da una citazione all'altra, si distingue il Liber de fide de apocryfis, l'unico a utilizzare la formula et ibi (ma anche et alibi) per segnalare una serie di citazioni tratte dal medesimo libro biblico.

L'unico caso in cui è possibile riscontrare singolari particolarità nelle formule di introduzione delle citazioni bibliche è rappresentato dal Tract. VIII, che, a differenza di tutti gli altri scritti, comincia direttamente e senza alcuna premessa con la citazione dei versetti iniziali del Salmo 3 seguiti da et reliqua; tale circostanza non si verifica mai negli altri trattati, nei quali, dopo una breve introduzione, sono citati via via solamente alcuni versetti del testo biblico preso in esame. Nel Tract. VIII una sola volta la citazione biblica è introdotta dalla formula sicut scribtum est, la più utilizzata nel complesso della raccolta, mentre per le rimanenti citazioni si trovano alcune formule non altrimenti attestate nel corpus di Würzburg, quali l'inconsueta espressione: $u t$ apostoli ad Efesios dictum est, e la formula tipicamente ciprianea: ponit et dicit, ${ }^{35}$ ben 4 volte si trova l'uso parentetico di inquid/ait all'interno della citazione, uso che negli altri scritti priscillianisti per quanto riguarda inquid in questa funzione è limitato a 5 attestazioni nel Liber apologeticus e nel Liber de fide de apocryfis, mentre non è attestato per quanto riguarda l'uso di ait; inoltre, nell'introdurre i passi di Rm 7, 14 e di 2 Pt 1, 20 l'apostolo Paolo è definito electum vas Christi, gentium doctor e Pietro beatissimus, apposizioni che si trovano solamente in questo testo. ${ }^{36}$

\section{Citazioni bibliche}

Negli scritti della raccolta si evidenzia la predilezione per alcuni libri biblici e per alcuni versetti scritturistici in particolare; in alcuni casi le citazioni ripetute concordano pressoché alla lettera, mentre in altri si possono rilevare delle discordanze, talora anche particolarmente significative. È necessario precisare che talvolta le discordanze si possono spiegare con il fatto che si tratta spesso di citazioni

35 Tract. VIII (CSEL 18.86.15; 87.7). Miodoński $(1889,28)$ contò 15 ricorrenze di questa formula nell'opera ciprianea, formula sconosciuta a Tertulliano e Lattanzio: Patient. 5; 9; 24; Zel. 13; Fort. 10; Mortal. 7 ; Domin. Orat. 6; Unit.Eccl. 12; Ep. 59.5; 59.13; 63.14; 69.1; 69.5; 73.19; 74.3; cf. inoltre Demetr. 17 e Domin.Orat. 2: posuerit et dixerit; Ep. 71.3: posuit dicens; epist. 17.1; 58.2; 63.10: ponit dicens; et alibi; per gli scritti pseudociprianei cf. Aleat. 3; 10 e Singul.Cler. 5.

36 Tract. VIII (CSEL 18.87 .6 e 8). 
fatte a memoria, per le quali anche l'esigenza della predicazione può avere di volta in volta svolto un ruolo importante nell'adattamento del dettato biblico. Ne è prova il fatto che anche nell'ambito dello stesso scritto la medesima citazione è riportata secondo versioni differenti. Ad esempio, nel Liber apologeticus il versetto di Eph 1, 23 è citato: omnia in omnibus adinpletur, e a poche righe di distanza: omnia et in omnibus adinpletur; ${ }^{37}$ per Ps 50, 5 nel Tract. X si legge: peccatum meum contra me est semper, ma anche: delictum meum contra me est semper. ${ }^{38}$

Fra le citazioni veterotestamentarie più frequenti si trovano i versetti di Bar 3 , 36-38 (hic est deus noster nec reputabitur alius absque eum) e di Sap 9, 15 (corpus quod corrumpitur adgravat animam et deprimit terrena inhabitatio sensum multa cogitantem) che sintetizzano efficacemente due temi fondamentali del pensiero priscillianista: 1'unicità di Dio e la dicotomia antropologica corpo-anima. A differenza del versetto sapienziale, riportato sempre secondo la medesima formulazione, ${ }^{39}$ le quattro citazioni di Bar 3, 36-38 presentano alcune differenze. ${ }^{40}$ Le citazioni del Liber apologeticus e del Tract. V sono perfettamente identiche: hic est deus noster nec reputabitur alius absque eum qui invenit omnem viam sapientiae et dedit eam Iacob puero suo et Istrahel dilecto suo; posthaec in terris visus est et cum hominibus conversatus est; mentre si evidenziano alcune differenze con quella presente nel Liber ad Damasum: hic est deus noster nec reputabitur alius absque eo qui accepit legem et dedit eam Iacob puero suo et Istrahel dilecto suo; posthaec in terris visus est et cum hominibus conversatus est; e quella del Liber de fide de apocryfis: hic est deus noster nec reputabitur alius absque eum qui ostendit viam disciplinae et dedit eam Iacob puero suo et Istrahel dilecto suo; posthaec in terris visus est et cum hominibus conversatus est.

Per il v. 36 si nota che la citazione del Liber ad Damasum è l'unica a riportare la forma corretta absque + ablativo (absque eo) contro absque eum degli altri testi, ${ }^{41}$ anche se non si può escludere che sia l'esito di una correzione da parte del copista. L'altra differenza sostanziale riguarda la proposizione relativa del v. 37, che presenta in questi scritti ben tre varianti: qui invenit omnem viam sapientiae / qui accepit legem / qui ostendit viam disciplinae. Il greco della Settanta recita: $\varepsilon \xi \varepsilon \tilde{v} \rho \varepsilon \pi \tilde{\alpha} \sigma \alpha v$

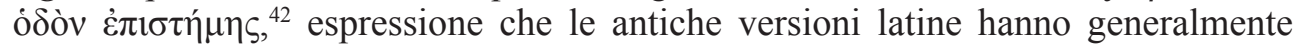
reso: invenit omnem viam disciplinae, con qualche lezione variante: viam scientiae / prudentiae / salutis / veritatis / doctrinae / iustitiae. ${ }^{43}$ Il Liber apologeticus e il Tract. V, nonostante rendano غ̇ंı

37 Lib. apol. I (CSEL 18.29.18 e 30.7). Cf. per Col 3,11: Lib. ad Dam. II (CSEL 18.37.23): omnia et in omnibus Christus, e Tract. V (CSEL 18.66.19): omnia in omnibus ... Christum.

38 Tract. X (CSEL 18.94.2; 96.17).

39 Tract. VI (CSEL 18.63.23); Tract. VI (CSEL 18.73.9); Tract. VII (CSEL 18.83.24).

40 Lib. apol. I (CSEL 18.5.18); Lib. ad Dam. II (CSEL 18.37.27); Lib. de fide de apocr. III (CSEL 18.49.10); Tract. $\mathrm{V}$ (CSEL 18.67.3). A queste si aggiunge la citazione del solo v. 36 in Lib. apol. I (CSEL 18.30.4).

${ }^{41}$ Così anche la citazione del solo v. 36 in Lib. apol. I (CSEL 18.30.4): ipse est deus noster nec alius reputabitur absque eum.

42 Ziegler $2013^{4}$ [1957], 462.

43 Sabatier 1751, 745. Viam disciplinae è nella maggior parte dei mss. della Vetus Latina e degli scrittori patristici: cf. Ambr. Fid. 1.3.28; 2.9.80; prudentiae si trova, ad esempio, in Cypr. Test. 2.6; Lact. Inst. 4.13.8; Maximin. C.Iud. 4.2; Aug. Civ. 18.33; scientiae in Hil. Trin. 4.42; 5.39; Amst. Quaest. 97.7; salutis in Actus Silvestri (ed. B. Mombritius, 520); veritatis in Somnium Neronis 12.2; doctrinae in Ps.Aug. solut. 43: 'qui invenit omnem viam disciplinae' (sive 'doctrinae', ut alii codices habent); iustitiae in Pelag. Trin. frg. 5.

44 Si veda una possibile allusione in Aug. C.Faust. 12.43: Hieremias dicit nimirum de sapientia: 'tradidit illam Iacob puero suo et Israhel electo suo... '. 
forma più vicina a quella del greco; così anche il Liber de fide de apocryfis che, pur riportando la lezione inconsueta ostendit, contro il comune invenit, non se ne discosta molto. È invece il Liber ad Damasum con qui accepit legem a presentare la variante più singolare che non trova corrispettivi né nel greco né nelle versioni latine, ma che potrebbe rappresentare un'interpretazione dell'espressione biblica viam disciplinae.

Un'altra citazione veterotestamentaria interessante è quella di Is 3,12 , presente in due trattati priscillianisti. ${ }^{45}$ Nel Tract. VII si legge: audi, populus meus, qui beatificant vos seducunt vos et semitas pedum vestrorum conturbant; mentre nel Tract. X: audi me, populus meus, quoniam sicut exactores vestri circumscribunt vos, ita et qui beatificant vos seducunt vos, ut semitas pedum vestrorum conturbent. In entrambe le citazioni compare all' inizio l'imperativo audi, ampliato in Tract. X in audi me, che non è altrimenti attestato come parte della citazione né nella Settanta né nella Vetus. ${ }^{46}$ Questa formula è impiegata molto spesso nell'antico Testamento per richiamare l'attenzione dell'uditorio, come esemplificano i Pss 49, 7 e 80, 9:

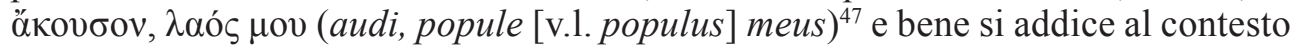
comunitario in cui i due sermoni furono predicati. Di contro a questa singolare analogia, le due citazioni presentano alcune differenze. La citazione del Tract. VII è abbreviata e riporta solamente la seconda parte del testo greco, ${ }^{48}$ secondo una formulazione che è conforme al testo che impiegavano usualmente gli autori cristiani latini del IV secolo, come ad esempio Rufino e Gerolamo. ${ }^{49}$ La citazione del Tract. X invece, più ampia ancorché non completa, introduce non solo una proposizione causale (quoniam) dove il greco ha una coordinata, ma anche una comparazione sicut... ita et... e una finale (ut... conturbent), che non hanno riscontro nel testo biblico della Settanta né in altri esegeti antichi. In entrambi gli scritti dunque il testo biblico presenta l'aggiunta originale dell'imperativo audi, ma per il resto le due citazioni presentano significative differenze.

Tra le citazioni neotestamentarie, alcuni versetti ricorrenti denotano il profondo interesse dei priscillianisti per le Scritture, ${ }^{50}$ che vanno attentamente indagate (Jo 5,39 ) e necessitano di interpretazione (2 Pt 1, 20). Il monito giovanneo Scrutate Scripturas ricorre in tutto quattro volte nel Liber apologeticus e nel Liber de fide de apocryfis: nella prima apologia si tratta di due chiare allusioni, mentre nello scritto in difesa degli apocrifi alle due esplicite citazioni si affianca anche la menzione del diligens scripturarum scrutator. ${ }^{51} \mathrm{Il}$ versetto di $2 \mathrm{Pt} 1,20$, non molto citato dagli antichi autori cristiani, ricorre invece tre volte nel corpus priscillianista, ma non

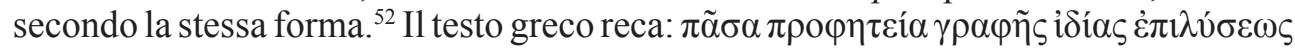

\footnotetext{
45 Tract. VII (CSEL 18.84.20); Tract. X (CSEL 18.95.8).

46 Ziegler 2013 ${ }^{4}$ [1939], 133; Gryson 1987-1993, 120-121.

47 La formula audi me è molto frequente nelle Scritture, cf. Prv 5, 7; 7, 24; Iob 15, 17; 33, 31; 33, 33; Is 48, 12; etc.

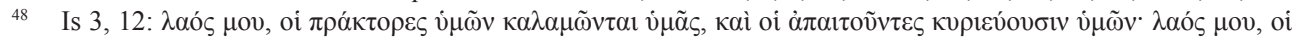

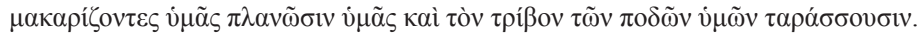

49 Cf. ad es. Orig.-Rufin. Hom. in Gen. 10.1: timeo enim illud, quod scriptum est: 'populus meus, qui beatificant vos, seducunt vos, et semitas pedum vestrorum conturbant'; Hier. a.Iovin. 2.37: unde per Isaiam loquitur deus: 'populus meus, qui vos beatificant, seducunt vos, et semitas pedum vestrorum conturbant'.

50 Vollmann 1974, 527: "Priscillianus Interesse galt nahezu ausschliesslich der scriptura".

51 Lib. apol. I (CSEL 18.12.20; 13.23); Lib. de fide de apocr. III (CSEL 18.47.25; 51.13; 45.23).

52 Lib. apol. I (CSEL 18.9.26): omnis profetia interpraetatione indiget; Tract. VI (CSEL 18.69.10): omnis scribtura interpraetationem indiget; Tract. VIII (CSEL 18.87.9): omnis profetia vel scribtura interpraetationem indiget.
} 


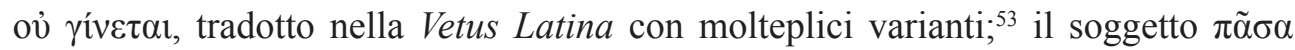
$\pi \rho о \varphi \eta \tau \varepsilon i ́ \alpha \gamma \rho \alpha \varphi \tilde{\eta} \varsigma$, interpretato variamente con prophetiae scriptura, prophetia vel scriptura o solamente scriptura o prophetia, è reso nel Liber apologeticus: omnis profetia, nel Tract. VI: omnis scribtura e nel Tract. VIII: omnis profetia vel scribtura; per il seguito la citazione, a parte l'oscillazione tra il corretto ablativo interpraetatione e l'accusativo interpraetationem, pure attestato in qualche antico manoscritto biblico, ${ }^{54}$ coincide con la versione dell'Ambrosiaster, di Bachiario e di un'opera falsamente attribuita a Mario Vittorino. ${ }^{55}$

Altri versetti citati frequentemente sono quelli desunti dalle epistole paoline, la cui predilezione da parte di Priscilliano è testimoniata dai Canones in Pauli epistolas. Ad esempio, è frequente l'immagine dei fedeli che battezzati in Cristo si sono rivestiti di Cristo, derivata da Gal 3, 27 (Lib. apol., Lib. ad Dam., Tract. V e VII), ${ }^{56}$ e che hanno appeso sulla croce la loro carne con le sue passioni e i suoi desideri (Tract. V, VI, X). Quest'ultima immagine proviene dalla citazione di Col 3, 9 (VL: exuentes vos veterem hominem cum actibus eius) in cui è stata inglobata quella di Gal 5, 24 (et concupiscentiis) ${ }^{57}$ questa particolare citazione, comune a tutti e tre i tractatus, non è molto diffusa, ma si riscontra solo in Ilario, nel Commento al Cantico dei Cantici di Filone di Carpasia e nella sua traduzione latina ad opera di Epifanio Scolastico. ${ }^{58}$ Inoltre, le citazioni del Tract. V e del Tract. X presentano due singolari aggiunte, ovvero: exsuentes $<a$ vobis $>$ (a nobis in Tract. X, dove tutta la frase è espressa con la prima persona plurale) e cum <omnibus $>$ actibus .

Un'altra immagine assai cara a diversi trattati è la metafora bellica del cristiano munito delle armi spirituali; nei Tract. IV, IX e X si legge quattro volte l'espressione indutus fidei armis, ${ }^{59}$ mentre nel Liber apologeticus si legge induti armis dei, ${ }^{60}$ che

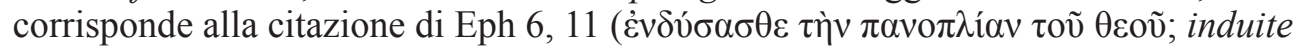
vos arma [v.1. armaturam] dei; cf. anche Eph 6, 13). La iunctura fidei arma è stata propiziata dal successivo v. 16 che menziona lo scudo della fede (fidei scutum) ed è attestata negli scritti di Cipriano, Mario Vittorino, Ambrosiaster, Leone Magno e nello Ps.Orienzo come variante di Eph 6, 11-13, ${ }^{61}$ anche se in alcuni casi si tratta

53 Thiele 1956-1969, 203.

54 Thiele 1956-1969, 203. Si tratta di un lezionario del VI secolo (Wolfenbüttel, Herzog August Bibliothek Weissenburg. 76 [Palimpsest]), un manoscritto veronese dei secc. VII/VIII (Verona, Biblioteca Capitolare X [8]), del ms. Harley 1772 (London, British Library) datato al IX secolo, a cui si aggiungono alcune edizioni della Bibbia di Isidoro datate ai secoli IX e X.

55 Amst. in I Tim. 2.4: dicit Petrus apostolus: omnis scriptura indiget interpretatione; Bachiar. fid. 6: omnis sermo interpretatione indiget; Ps.Marius Vict. De verbis scripturae 1 (PL 8, 1009D): omnis enim scriptura spiritu dei inspirata dicit apostolus Paulus quia indiget interpretatione.

56 Lib. apol. I (CSEL 18.5.2): baptizati in Christo induti Christum; Lib. ad Dam. II (CSEL 18.34.15): baptizati in Christo Christum induentes; Tract. V (CSEL 18.64.21): baptizati in Christo Christum induistis; Tract. VII (CSEL 18.83.15): baptizati in Christo Christum induentes.

57 Tract. V (CSEL 18.67.9): exuentes a vobis veterem hominem cum omnibus actibus et concupiscentiis suis; Tract. VI (CSEL 18.81.1): exsuentes veterem hominem cum omnibus actibus et concupiscentiis suis; Tract. X (CSEL 18.98.12): veterem a nobis hominem exui cum omnibus actibus et concupiscentiis suis.

58 Hil. In Psalm. 66.7; In Psalm. 118 samech 13: vetere homine cum vitiis et concupiscentiis eius exuto; Phil. Carp.

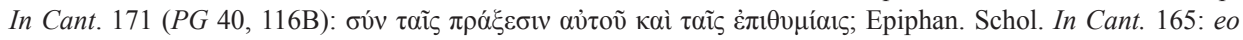
quod exuerint se vetere homine cum actibus et concupiscentiis suis.

59 Tract. IV (CSEL 18.61.4); Tract. IX (CSEL 18.90.6); Tract. X (CSEL 18.93.1 e 95.19).

60 Lib. apol. I (CSEL 18.15.5): secundum scribturas induti armis dei...

${ }_{61}$ Frede 1962-1964, 275-280; 294-303. Cypr. Epist. 10.2.2: armis fidei credentis armatos. Marius Vict. In Eph. 6.13: accipite arma fidei. Amst. Quaest. 126.14: suscepta arma fidei; Eph 6.11: nel testo di Eph 6, 11 cita correttamente armis dei, e nel commento afferma: arma dei fides est stabilis. Max. Taur. Serm. 61.3: haec sunt 
più di una espressione ormai consueta senza un diretto collegamento con il passo scritturistico.

Anche l'Epistola di Giacomo, la cui autorità e canonicità furono lungamente discusse, ha riscosso grande favore negli scritti priscillianisti, in cui ricorrono frequenti le citazioni di Jac 4, 1 e 4, 4, rispettivamente sulla lotta interna all'uomo scaturita dalle passioni che si agitano nelle sue membra, e sull'amicizia nei confronti del mondo che risulta essere inimicizia nei confronti di Dio. La citazione di Jac 4, 1 compare nel Tract. V e nel Tract. $\mathrm{X}^{62}$ secondo una forma singolare, che traduce

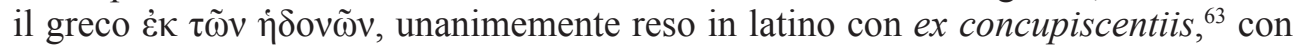
de voluntatibus: unde bella, unde rixae in vobis? nonne de voluntatibus vestris? Lo scambio voluntas/voluptas è certamente un comune caso di corruttela, ${ }^{64}$ ma la coincidenza con lo Speculum ps.agostiniano, che tanto spesso condivide singolari varianti del testo biblico con gli scritti priscillianisti, ${ }^{65} \mathrm{e} \mathrm{la} \mathrm{ricorrenza} \mathrm{del} \mathrm{medesimo}$ scambio anche in altre citazioni del corpus di Würzburg, ci induce a mantenere questa lezione. ${ }^{66}$ Ancora, i Tract. IV, IX e X condividono la singolare inserzione dell'aggettivo omnis, altrimenti non attestata, nella citazione di Jac 4, 4: omnis

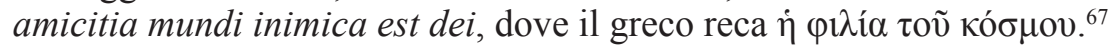

La citazione più frequente negli scritti della raccolta priscillianista con otto

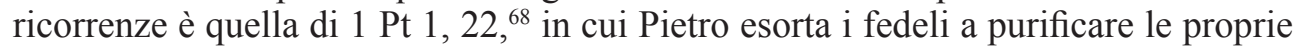
anime per obbedire alla fede; tale predilezione si spiega con il fatto che in essa si trovano indicati contemporaneamente il metodo e lo scopo della vita cristiana. ${ }^{69}$ Oltre a qualche piccola differenza tra le varie citazioni, ${ }^{70}$ è da notare che nel Liber apologeticus e nel Tract. IX il versetto è ampliato con l'inserzione < per spiritum $>.^{71}$ Questa aggiunta si trova anche nello Speculum ps.agostiniano, nello Ps.Vigilio e in Gilda ${ }^{72}$ questi tre testi concordano con le citazioni del corpus priscillianista anche per il resto della citazione, dove rendono ad obaudiendum fidei quello che nel testo

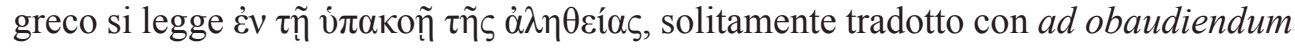

arma fidei nostrae. Leo M. Serm. 9.4:...totam ubique ecclesiam decet fidei arma corripere. Ps.Orient. Carm. 3.32: arma fidei.

62 Tract. V (CSEL 18.63.16); Tract. X (CSEL 18.96.19). Cf. anche Tract. IV (CSEL 18.58.26).

63 Thiele 1956-1969, 44. Cf. l'eccezione di Hier. Pelag. 2.19: unde bella et unde rixae inter vos? nonne ex voluptatibus, quae militant in membris vestris?

64 Svennung 1922, 133-136.

65 Ps.Aug. Spec. 101: Item Iacobus apostolus: Unde bella? Unde rixae in vobis? Nonne de voluntatibus vestris, quae militant in membris vestris? Cf. Veronese 2018, 254-263.

66 Anche nelle citazioni di 2 Pt 2, 10 e 1 Jo 2, 16, dove nel testo greco si legge غ̇ंı $\theta v \mu$ ía, la versione latina dei Tractatus reca voluntas. Cf. Veronese 2018, 253.

${ }_{67}$ Tract. IV (CSEL 18.57.7); Tract. IX (CSEL 18.90.16); Tract. X (CSEL 18.94.15).

68 Lib. apol. I (CSEL 18.4.6): castificavimus animas nostras ad obaudiendum fidei per spiritum; Tract. IV (CSEL 18.58.2 e 25): ad obaudiendum nos fidei ... castificate animas vestras deo; Tract. IV (CSEL 18.59.6): castificati corpore et spiritu caritatem in dilectione simplicem ex corde vero; Tract. V (CSEL 18.67.8): castificate animas vestras ad obaudiendum fidei; Tract. VI (CSEL 18.80.2): castificate animas vestras ad obaudiendum; Tract. VII (CSEL 18.85.5): castificate animas vestras ad obaudiendum fidei; Tract. IX (CSEL 18.90.7): castificate animas vestras ad obaudiendum per spiritum... caritatem in fraternitate simplicem in gloriam divinae dilectionis adhibete.

69 Babut 1909, 111.

70 Tract. IV (CSEL 18.59.6): caritatem in dilectione, e Tract. IX (CSEL 18.90.9): caritatem in fraternitate.

71 Lib. apol. I (CSEL 18.4.16); Tract. IX (CSEL 18.90.7).

72 Ps.Aug. Spec. 78: Animas itaque vestras castificate ad oboediendum fidei per spiritum; Ps. Vigil. C.Varimad. 3.58: animas vestras per spiritum castificate ad obedientiam fidei; Gilda De excidio et conquestu Britanniae 106: animas vestras castificate ad oboediendum fidei per spiritum in caritate in fraternitate ex corde vero invicem diligentes perseveranter. 
veritatis o caritatis $;{ }^{73}$ tale versione è dovuta a un probabile influsso di Rm 1, 5: عi $\varsigma$

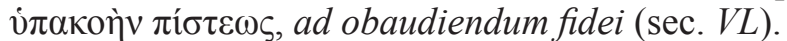

L'esortazione petrina a offrire a Dio sacrifici spirituali di $1 \mathrm{Pt} 2,5$ è presente due volte nei trattati; nel Tract. V si legge: et vos tamquam lapides vivi aedificamini in domos spiritales, offerentes hostias inmaculatas acceptabiles deo; mentre nel Tract. VI si presenta ridotta e con qualche modifica: et offerte vos tamquam spiritales hostias, acceptabile sacrificium placens deo. ${ }^{74} \mathrm{Se}$ in quest'ultimo passo, seguendo il precetto petrino ( $\pi v \varepsilon v \mu \alpha \tau \iota \kappa \alpha ̀ \varsigma ~ \theta v \sigma i ́ \alpha \varsigma)$, si parla di vittime spirituali, nel Tract. V si parla di hostias inmaculatas, una lezione del tutto singolare, che Thiele propone di intendere come una "dublette". ${ }^{75}$

Significativo è il caso del passo di Apc 16,13-14, sulle cui differenze si basò Sittl per scardinare la tesi di Schepss, il quale era convinto che ovunque nella raccolta fosse stata utilizzata la medesima versione della Scrittura. ${ }^{76}$ Le citazioni si leggono nel Liber apologeticus: vidi de ore draconis et de ore bestiae et de ore pseudoprofetae spiritus tres inmundos sicut ranas et hi sunt spiritus daemoniorum, qui facientes signa procedunt ad conturbandos reges orbis terrae; e nel Tract. X: vidi tres spiritus inmundos tamquam tres ranas exeuntes de ore pseudoprofetae et de ore bestiae et hii sunt tres spiritus daemoniorum qui procedunt ad conturbandos reges terrae. ${ }^{77}$

La versione del Liber apologeticus è esattamente conforme al testo greco ([13]

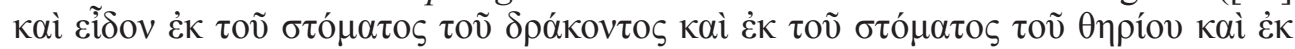

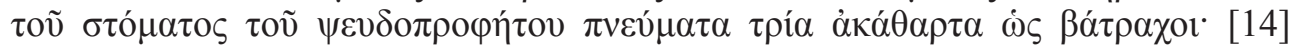

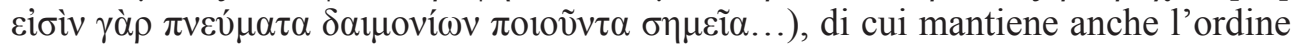
delle parole; nel Tract. X, invece, si notano l'inversione dei termini del v. 13 con la soppressione di de ore draconis, la ripetizione del numero tre (tres spiritus... tres ranas... tres spiritus), l'aggiunta del participio exeuntes e l'eliminazione di facientes signa al v. 14. Queste variazioni, tuttavia, si possono spiegare in funzione del contesto della predicazione al popolo: così si giustificano l'avere anticipato la menzione dei tre spiriti immondi e l'aggiunta di exeuntes -comunque non estranea alla tradizione biblico-patristica essendo comune a Primasio e presente anche in un manoscritto biblico greco ( $\dot{\varepsilon} \xi \varepsilon \rho \chi o ́ \mu \varepsilon v \alpha)$ e in alcuni manoscritti latini, anche coniugato all'infinito exire- ${ }^{78}$ che facilita la comprensione del passo, mentre risulta superflua al contesto omiletico l'espressione facientes signa.

Ma era sfuggito a Sittl il fatto che in questo passo più delle differenze colpisce la stretta analogia nella parte finale dove si legge: ad conturbandos reges orbis terrae, contro la unanime lezione del testo giovanneo che narra che gli spiriti "si recano presso i re di tutta la terra per radunarli insieme in battaglia nel gran

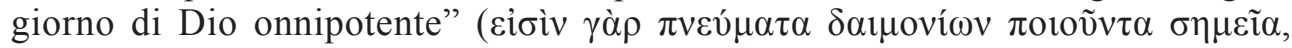

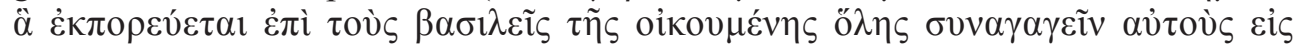

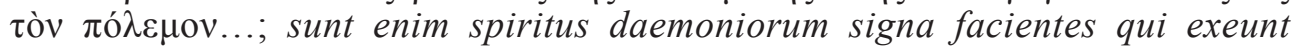

73 Thiele 1956-1969, 91.

74 Tract. V (CSEL 18.68.4); Tract. VI (CSEL 18.80.3)

75 Thiele 1956-1969, 100-102. Solamente in Leone Magno, nel commento che egli propone al versetto, ma non nella citazione che è corretta, si parla di inmaculatas pietatis hostias de altari cordis offerre, cf. Leo M. Tract. 4.1.

76 Sittl 1889, 44.

77 Lib. apol. I (CSEL 18.13.10-13); Tract. X (CSEL 18.96.13-16).

78 Gryson 2000-2003, 574. Primas. In Apoc. 4; il participio $\dot{\varepsilon} \xi \varepsilon \rho \chi \dot{\mu} \mu \varepsilon v \alpha$ si trova in un ms. del XV sec. custodito ad Atene (2091 Aland), mentre exeuntes in un ms. di Cava dei Tirreni $(C)$ e in due mss. di Madrid $\left(\Sigma^{\mathrm{T}}\right.$ e $\left.\Sigma^{\mathrm{O}}\right)$; la forma exire era attestata nel Codex Demidovianus, ora perduto. 
[v.1. procedunt] ad reges totius terrae contrahere [v.1. congregare] illos in proelium...). ${ }^{79}$ Sulla singolare citazione giovannea dell'autore priscillianista, di cui non ho trovato altra attestazione, può avere influito il testo dei Salmi, in particolare l'immagine dei re che nel suo sdegno il Messia sconvolgerà, secondo

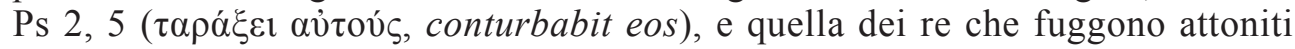

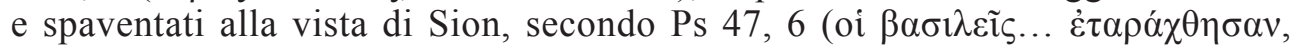
reges... conturbati sunt).

La perfetta coincidenza di questa versione di Apc 16, 14, non altrimenti attestata, sia nel Liber apologeticus sia nel Tract. X, che pure non modifica sostanzialmente il significato del passo di Apocalisse ma ne costituisce piuttosto una sorta di commento esegetico, è più significativa delle divergenze che caratterizzano le due citazioni.

\section{Conclusioni}

Una delle prove che gli studiosi hanno chiamato in causa pro e contra la tesi dell'unitarietà d'autore degli scritti della raccolta priscillianista di Würzburg deriva dal confronto delle citazioni scritturistiche dei trattati. L'analisi qui condotta ha considerato non solo analogie e differenze individuate nei versetti biblici citati più volte all'interno del corpus, ma anche le formule di introduzione delle citazioni stesse.

Per quanto riguarda le citazioni bibliche ripetute non molte sono le differenze sostanziali, mentre sono talora più significative le analogie in lezioni singolari che non trovano altre attestazioni. Nel complesso se ne deduce che gli scritti della raccolta gravitano nella medesima orbita culturale, con l'unica eccezione del Tract. VIII che pare fare causa a sé; l'analisi evidenzia alcune tendenze che contraddistinguono i Tract. V e VII dai rimanenti Tract. IV, VI, IX e X. Più complicati risultano invece i rapporti tra i Tractatus e le tre apologie iniziali, che non consentono conclusioni certe e univoche. In conclusione, il contributo che questa indagine può apportare alla definizione della questione sull'unitarietà o meno del corpus priscillianista va considerato utile ma va adoperato con prudenza e solo nel quadro di un'indagine complessiva che rivaluti tra l'altro la lingua e lo stile degli scritti a partire da una nuova e affidabile edizione critica.

\section{Bibliografia}

Babut, E.-Ch. (1909): Priscillien et le Priscillianisme (=Bibliothèque de l'École des Hautes Études; Sciences Historiques et Philologiques 169), Paris.

Bardy, G.

(1936): "Priscillien”, [in] Dictionnaire de Théologie Catholique 13.1, Paris, 391-400.

(1946): “Tractare, tractatus (précher, prédication, célébrer l'eucharistie)", Recherches de Science Religieuse 32, 211-236.

Burrus, V.

79 Gryson 2000-2003, 574-575. 
(1995): The Making of a Heretic. Gender, Authority, and the Priscillianist Controversy, Berkeley-Los Angeles-London.

(1997): "Priscillianist duplicity reconsidered", [in] E. A. Livingstone (ed.), Papers presented at the Twelfth International Conference on Patristic Studies held in Oxford 1995 (=Studia Patristica 33), Leuven, 401-406.

Chadwick, H. (1976): Priscillian of Avila. The Occult and the Charismatic in the Early Church, Oxford.

CLA: E. A. Lowe (ed.), Codices latini antiquiores. A palaeographical guide to Latin manuscripts prior to the ninth century, Oxford, 1934-1966.

Conti, M. (ed. \& trans.), (2010): Priscillian of Avila. The complete Works, Oxford.

CPL: E. Dekkers (ed.), Clavis Patrum Latinorum, Turnhout, $1995^{3}$.

CPPM: J. Machielsen (ed.), Clavis Patristica pseudepigraphorum Medii Aevi, Turnhout, 1990-2004.

CSEL: Corpus Scriptorum Ecclesiasticorum Latinorum, Wien, 1866- (Berlin dal 2012).

D’Alés, A. (1936): Priscillien et l'Espagne chrétienne à la fin du IV siècle, Paris.

De Bruyne, D. (1914-1919): "Études sur les origines de la Vulgate en Espagne”, Revue Bénédictine 31, 373-401 (https://doi.org/10.1484/J.RB.4.01819).

Dierich, J. (1897): Die Quellen zur Geschichte Priszillians, Breslau.

Fischer, B. (1963): "Bibelausgaben des frühen Mittelalters", [in] La Bibbia nell'Alto Medioevo (=Settimane di studi del Centro italiano di Studi sull'Alto Medioevo X), Spoleto, 519-600.

Fontaine, J. (1981): "Panorama espiritual del Occidente peninsular en los siglos $\mathrm{IV}^{\circ} \mathrm{y} \mathrm{V}^{\circ}$. Por una nueva problemática del priscilianismo", [in] Primera reunión gallega de estudios clásicos (Santiago-Pontevedra, 2-4 julio 1979), Santiago de Compostela, 185-209 [rist. in J. Fontaine, Culture et spiritualité en Espagne du IV au VII siècle (=Collected studies series 234), London, 1986, stessa numerazione].

Frede, H. J. (ed.), (1962-1964): Vetus Latina. Die Reste der altlateinischen Bibel, 24.1. Epistula ad Ephesios, Freiburg i.B.

Gryson, R. (ed.)

(1987-1993): Vetus Latina. Die Reste der altlateinischen Bibel, 12.1. Esaias, Freiburg i.B. (2000-2003): Vetus Latina. Die Reste der altlateinischen Bibel, 26.2. Apocalypsis Johannis, Freiburg i.B.

Hilgenfeld, A. (1892): "Priscillianus und seine neuentdeckten Schriften", Zeitschrift für Wissenschaftliche Theologie 35, 1-85.

Kraus, F. X. (1891): "Corpus scriptorum ecclesiasticorum latinorum editum consilio et impensis academiae litterarum Caesareae Vindobonensis. Vol. XV: Commodiani Carmina ex recensione Bernhardi Dombart. XXIV u. 250 S. $8^{\circ}$. Wien, Gerold's Sohn, 1887. - Vol. XVIII: Priscilliani quae supersunt recensuit Georgius Schepss. XLVI u. 223 S. $8^{\circ}$. Wien u. Prag, F. Tempsky, 1889", Literarische Rundschau 17, 110-111.

Mercati, G. (1901): "I due Trattati al popolo di Priscilliano", [in] G. Mercati, Note di letteratura cristiana antica (=Studi e Testi 5), Roma, 127-136.

Merkle, S. (1896): “Der Streit über Priscillian”, Theologische Quartalschrift 78, 630-649.

Michael, E. (1892): "Priscillian und die neueste Kritik", Zeitschrift für katholische Theologie 16/4, 692-706.

Miodoński, A. (1889): Anonymus, Adversus aleatores (gegen das Hazardspiel) und die Briefe an Cyprian, Lucian, Celerinus und an den karthaginiensischen Klerus (Cypr. epist. 8, 21 24), Erlangen-Leipzig. 
Mohrmann, C. (1961): Études sur le latin des Chrétiens, I (=Storia e Letteratura. Raccolta di Studi e Testi 65), Roma.

Morin, G. (1913): "Pro Instantio. Contre l'attribution à Priscillien des opuscules des manuscripts de Würzburg”, Revue Bénédictine 30, 153-173 (https://doi.org/10.1484/J. RB.4.01769).

Paret, F. (1891): Priscillianus: ein Reformator des vierten Jahrhunderts, Würzburg.

PL: J.-P. Migne (ed.), Patrologiae cursus completus, series Latina, Paris, 1844-1864.

Puech, A. (1912): "Les origines du Priscillianisme et l'orthodoxie de Priscillien", Bulletin d'ancienne Littérature et d'Archéologie Chrétienne 2, 81-95; 161-213.

Sabatier, P. (1751): Bibliorum sacrorum Latinae versiones antiquae seu vetus Italica et caeterae quaecumque in codicibus mss. et antiquorum libris reperiri potuerunt, II, Reims [ripr. facs. Turnhout 1981].

Sanchez, S. J. G.

(2009): Priscillien, un chrétien non conformiste. Doctrine et pratique du priscillianisme $d u I V^{e}$ au $V I^{e}$ siècle (=Théologie historique 120), Paris.

(2013): "Étude des différentes rédactions du Liber apologeticus du codex de Wurtzbourg attribué à Priscillien", Revista Catalana de Teologia 38/1, 209-229.

Schatz, W. (1957): Studien zur Geschichte und Vorstellungswelt des frühen abendländischen Mönchtums, Masc. Diss., Freiburg i.B.

Schepss, G.

(1886a): Priscillian. Ein neuaufgefundener lateinischer Schriftsteller des vierten Jahrhunderts, Würzburg.

(1886b): "Die Sprache Priscillians", Archiv für lateinische Lexicographie 3, 309-328.

(1889): Priscilliani quae supersunt maximam partem nuper detexit adiectisque commentariis criticis et indicibus primus edidit Georgius Schepss, accedit Orosii Commonitorium de errore priscillianistarum et origenistarum (=Corpus Scriptorum Ecclesiasticorum Latinorum 18), Pragae-Vindobonae-Lipsiae.

(1893): "Pro Priscilliano", Wiener Studien 15, 128-147.

Sittl, K.

(1889): “G. Schepss, Priscillian. Ein neuaufgefundener lateinischer Schriftsteller des vierten Jahrhunderts, Würzburg (Stuber) 1886", Jahresbericht über die Fortschritte der classischen Alterthumswissenschaft II. Abt., 59, 44-45.

(1891): “Georg Schepss, Die Sprache Priscillians, Arch. Bd. III S. 309-28”, Jahresbericht über die Fortschritte der classischen Alterthumswissenschaft II. Abt., 68, 267-269.

Svennung, J. (1922): Orosiana. Syntaktische semasiologische und kritische Studien zu Orosius (=Uppsala Universitets Årsskrift. Filosofi, Språkvetenskap och historiska vetenskaper 5), Uppsala.

Thiele, W. (ed.), (1956-1969): Vetus Latina. Die Reste der altlateinischen Bibel, 26.1. Epistulae catholicae, Freiburg.

Thurn, H. (1984): Die Pergamenthandschriften der ehemaligen Dombibliothek, 3/1, Wiesbaden.

Veronese, $\mathrm{M}$.

(2003): "Le citazioni del De Trinitate di Ilario nella raccolta attribuita a Priscilliano", Vetera Christianorum 40, 133-157.

(2004): “Il digiuno di Giosuè: errore di citazione o corruzione priscillianista?", Auctores Nostri 1, 201-214.

(2013): “Ancora sul De Trinitate di Ilario e i trattati priscillianisti. Note critiche", Vetera Christianorum 50, 313-327. 
(2018): Dilibatio et massa. La Scriptura nella raccolta di Würzburg attribuita a Priscilliano (=Auctores Nostri 20), Bari.

Vollmann, B. (1974): "Priscillianus", [in] Paulys Real-Encyclopädie der classischen Altertumswissenschaft, Suppl. 14, Stuttgart, 485-559.

Ziegler, J. (ed.)

(1983³ [1939]): Septuaginta. Vetus Testamentum graecum, XIV: Isaias, Göttingen.

(20134 [1957]): Septuaginta. Vetus Testamentum graecum, XV: Jeremias, Baruch, Threni, Epistula Jeremiae, Göttingen. 